\title{
Hyperthermic intraperitoneal chemotherapy (HIPEC) on the electrolytes changes and nefropaty
}

\author{
Aslan Bilge ${ }^{*}$, Aydın Feray ${ }^{2}$ and Güngör Tayfun ${ }^{3}$ \\ ${ }^{1}$ Anesthesiologist, Zekai Tahir Burak Training and Research Hospital, Anesthesia Clinic Department, 06050, Turkey \\ ${ }^{2}$ General Surgery, Zekai Tahir Burak Training and Research Hospital, Turkey \\ ${ }^{3}$ Gynecological Oncologist, Zekai Tahir Burak Training and Research Hospital Oncology Gynecology Clinic Department Chief, Turkey
}

\begin{abstract}
Objective: Hyperthermia intraperitoneal chemoprophylaxis (HIPEC) is a treatment of peritoneal carcinomatosis with potential iatrogenic. This study was designed to define the effects of HIPEC on renal functions, electrolytes, nefropaty.

Methods: We retrospectively reviewed the perioperative care of 20 patients. After cytoreductive surgery (CS), residual tumors were not present in all cases, or were less than or equal to $3 \mathrm{~mm}$ (measured in size). Cisplatin $50 \mathrm{mg} / \mathrm{m}^{2}$ plus doxorubicin $15 \mathrm{mg} / \mathrm{m}^{2}$ was used. This is a retrospective study evaluating patients receiving cisplatin with doxorubicin during HIPEC.

Findings: 20 patients were identified. Based on the RIFLE classification, patients (2.0\%) developed acute renal damage (AKI) with HIPEC after cisplatin. The other patient had renal damage. Comparable mean creatinine levels were observed at baseline and on postoperative 21th day after HIPEC (p > 0.05). The incidence of hypomagnesaemia increased to $10 \%(\mathrm{p}=0.02)$ on the 5 th day and to $5 \%$ on the 21 th day $(\mathrm{p}>0.05)$ after HIPEC.
\end{abstract}

Conclusion: Nephrotoxicity and permanent renal dysfunction may rarely occur in cisplatin therapy with HIPEC. We should closely monitor postoperative Mg levels in patients who have undergone HIPEC administration. Doxsorubicin may have cardiotoxic side effects.

\section{Introduction}

All patients received HIPEC during general anesthesia. Standard induction was $8 \mathrm{mg} / \mathrm{kg}$ pentothal, $1 \mu / \mathrm{kg}$ fentanyl, and $6 \mathrm{mg} / \mathrm{kg}$ rocuronium intravenously. In the case of all patients in the place were opened as MAC:1 sevolurane and 40\% Oxygen $+60 \%$ nitrogen was given. TOF and BIS moniterisations were standardized. After debalking operation, HIPEC was performed using a $0.9 \%$ isotonic solution of cisplatin + doxarubicin for 40 minutes at $41-42^{\circ} \mathrm{C}$ [1-4]. All patients underwent open perfusion. Metabolic, electrolyte and hemodynamic changes were noted during chemo-fusion, as well as postoperative metabolic, electrolyte and hemodynamic changes recorded on days 3 , 5 and 21 [5-8].

\section{Methods}

We retrospectively reviewed the perioperative care of 20 patients. After cytoreductive surgery (CS), residual tumors were not present in all cases, or were less than or equal to $3 \mathrm{~mm}$ (measured in size). Cisplatin $50 \mathrm{mg} / \mathrm{m}^{2}$ plus doxorubicin $15 \mathrm{mg} / \mathrm{m}^{2}$ was used. This is a retrospective study evaluating patients receiving cisplatin with doxorubicin during HIPEC.

Comorbidities and effects of nephrotoxic drugs were found. Renal function parameters, including serum magnesium levels, were also collected on preoperative and postoperative creatinine levels and on days 1, 5 and 21 post HIPEC. Perioperative urine output (UO) was also recorded.

\section{Results}

From January 2010 to January 2016, 20 patients were treated. Chemofusion with $0.9 \%$ isotonic resulted in transient significant hyponatremia and metabolic acidosis. While major morbidity occurred in $5 \%$ of patients, there was no mortality for 6 months $[9,10]$. Chemical interaction with doxorubicin led to mild hepatic toxicity but not significant. Hipomagnesemia was found to be significant when serum magnesium levels were below $0.7 \mathrm{mmol} / \mathrm{L}$. On the 2 nd day after operation in one patient in V4, V5, V6 T negative. T negativity

Table 1. Demographic Datas

\begin{tabular}{|c|c|c|c|}
\hline & All patients $n=\mathbf{2 0}$ & Patients with RIF & $p$ Value \\
\hline Female, $\%$ & $20(94.3 \%)$ & $2(10 \%)$ & \\
\hline Age (years) & $49.6 \pm 10.1$ & $47.50 \pm 10$ & 0.65 \\
\hline Weight (kg) & $70.9 \pm 14.7$ & $75.1 \pm 29$ & 0.01 \\
\hline BSA $\left(\mathbf{m}^{2}\right)$ & $1.75 \pm 0.6$ & $2.06 \pm 0.6$ & 0.042 \\
\hline Comorbidities & - & - & - \\
\hline Diabetes mellitus & $4(20 \%)$ & $1(50 \%)$ & 0.43 \\
\hline Hypertension & $5(25 \%)$ & $2(100 \%)$ & 0.045 \\
\hline
\end{tabular}

${ }^{\star}$ Correspondence to: Aslan Bilge, Anesthesiologist, Zekai Tahir Burak Training and Research Hospital, Anesthesia Clinic Department, Talatpaşa Bulvarı, 06050, Ankara/Turkey, Tel: +90 312 3065184; E-mail: drbilgeaslan@hotmail.com

Key words: Peritoneal Carcinomatosis, HIPEC, cisplatin, doxorubicin, electrolytes Received: March 05, 2018; Accepted: March 16, 2018; Published: March 20, 2018 
Table 2. Electrolytes data

\begin{tabular}{|l|l|l|}
\hline & Pre-HIPEC $(\mathbf{n}=\mathbf{2 0})$ & Post-HIPEC $(\mathbf{n}=\mathbf{2 0})$ \\
\hline Sodium $(\mathrm{mmol} / \mathrm{L})$ & 135 & 135 \\
\hline Potassium $(\mathrm{mmol} / \mathrm{L})$ & 4.2 & 4.3 \\
\hline Chloride(mmol/L) & 108 & 104 \\
\hline Calcium & 8.1 & 7.1 \\
\hline Bicarbonate(mmol/L) & 19 & 21 \\
\hline Creatinine & 0.39 & 0.45 \\
\hline BUN $(\mathrm{mg} / \mathrm{dL})$ & 19 & 21 \\
\hline GFR $\left(\mathrm{ml} / \mathrm{min} / 1.73 \mathrm{~m}^{2}\right)$ & $90.50 \pm 16.00$ & $80.13 \pm 23.02$ \\
\hline $\mathrm{Mg}(1.2-2.6 \mathrm{mg} / \mathrm{dL})$ & $2.4 \pm 0.37$ & $1.78 \pm 0.50$ \\
\hline PH & 7.42 & 7.33 \\
\hline
\end{tabular}

Table 3. RIFLE classification for GFR: glomerular filtration rate; UO: urine output; ESRD end-stage renal disease.

\begin{tabular}{|l|l|l|}
\hline Category & GFR criteria & $\mathrm{UO}$ criteria \\
\hline Risk (R) & $\begin{array}{l}\text { Increased creatinine level } \times 1.5 \text { or GFR } \\
\text { decrease }>25 \%\end{array}$ & $\mathrm{UO}<0.5 \mathrm{~mL} / \mathrm{kg} / \mathrm{h} \times 6 \mathrm{~h}$ \\
\hline Injury (I) & $\begin{array}{l}\text { Increased creatinine level } \times 2 \text { or GFR } \\
\text { decrease }>50 \%\end{array}$ & $\mathrm{UO}<0.5 \mathrm{~mL} / \mathrm{kg} / \mathrm{h} \times 12 \mathrm{~h}$ \\
\hline Failure (F) & $\begin{array}{l}\text { Increased creatinine level } \times 3 \text { or GFR } \\
\text { decrease }>75 \% \text { or creatinine level }>4 \mathrm{mg} / \mathrm{dL}\end{array}$ & $\begin{array}{l}\mathrm{UO}<0.3 \mathrm{~mL} / \mathrm{kg} / \mathrm{h} \times 24 \mathrm{~h} \text { or } \\
\text { anuria } \times 12 \mathrm{~h}\end{array}$ \\
\hline Loss (L) & $\begin{array}{l}\text { Persistent acute renal failure or complete } \\
\text { loss of renal function for }>4 \text { weeks }\end{array}$ & \\
\hline ESRD (E) & ESRD for $>3$ months & \\
\hline
\end{tabular}

improved within a few days. Troponin I and CK-MB never elevated in this patient. Transient hypotension developed in two patients. Doxsorubicin may have cardiotoxic side effects.

\section{Conclusion}

Cytoreduction with HIPEC using high dose cisplatin and leads to manageable metabolic and electrolyte disturbances, rarely nephrotoxicity and frequent mild hepatic toxicity without noticable impact on postoperative morbidity. Doxsorubicin may have cardiotoxic side effects.

\section{References}

1. Armstrong DK, Bundy B, Wenzel L (2006) Intraperitoneal cisplatin and paclitaxel in ovarian cancer. $N$ Engl J Med 354: 34-43. [Crossref]

2. Flessner MF (2005) The transport barrier in intraperitoneal therapy. Am J Physiol Renal Physiol 288: 433-442. [Crossref]

3. Tannock IF, Lee CM, Tunggal JK (2002) Limited penetration of anticancer drugs through tumor tissue: A potential cause of resistance of solid tumors to chemotherapy. Clin Cancer Res 8: 878-884. [Crossref]

4. Verwaal VJ, van Ruth S, de Bree E (2003) Randomized trial of cytoreduction and hyperthermic intraperitoneal chemotherapy versus systemic chemotherapy and palliative surgery in patients with peritoneal carcinomatosis of colorectal cancer. $J$ Clin Oncol 21: 3737-3743. [Crossref]

5. Yan TD, Black D, Savady R (2006) Systematic review on the efficacy of cytoreductive surgery combined with perioperative intraperitoneal chemotherapy for peritoneal carcinomatosis from colorectal carcinoma. J Clin Oncol 24: 4011-4019. [Crossref]

6. Van Ruth S, Mathot RAA, Sparidans RW (2004) Population pharmacokinetics and pharmacodynamics of mitomycin during intraoperative hyperthermic intraperitoneal chemotherapy. Clin Pharmacokin 43: 131-143. [Crossref]

7. Chau I, Cunningham D (2006) Adjuvant therapy in colon cancer - what, when and how? Ann Oncol 17: 1347-1359.

8. Elkas JC, Winter WE, Chernofsky MR (2007) A phase I trial of oxaliplatin and topotecan in recurrent ovarian carcinoma. Gynecol Oncol 104: 422-427. [Crossref]

9. Zorzi D, Laurent A, Pawlik TM (2007) Chemotherapy-associated hepatotoxicity and surgery for colorectal liver metastases. Br J Surg 94:274-286. [Crossref]

10. Stephens AD, Alderman R, Chang D (1999) Morbidity and mortality analysis of 200 treatments with cytoreductive surgery and hyperthermic intraoperative intraperitoneal chemotherapy using the Coliseum technique. Ann Surg Oncol 6: 790-796. [Crossref]

Copyright: (C2018 Bilge A. This is an open-access article distributed under the terms of the Creative Commons Attribution License, which permits unrestricted use, distribution, and reproduction in any medium, provided the original author and source are credited. 